\title{
Cuantificación del Riesgo en la Teoría de Carteras
}

\author{
Juliano Lima Pinheiro ${ }^{1}$
}

\section{Resumen}

En este artículo elaboraremos un marco conceptual con un resumen de los principales conceptos respecto a la cuantificación del riesgo financiero según la Teoría de Carteras, distinguiendo entre las diferentes acepciones existentes en la literatura económica y examinando sus intereraciones con el mercado financiero.

Palabras-clave: Riesgo, Riesgo Financiero, Operaciones Financieras, Mercado Financiero.

\section{Resumo}

Neste artigo desenvolveremos um marco conceitual com um resumo dos principais conceitos a respeito da quantificação do risco financeiro segundo a Teoria de Carteiras, distinguindo-se entre as diferentes acepções existentes na literatura econômica e examinando suas interligações com o mercado financeiro.

Palavras-chave: Risco, Risco Financeiro, Operações Financeiras, Mercado Financeiro.

\begin{abstract}
In this paper, a conceptual framework is built up with the main definitions related to financial risk quantification, according to the Portfolio Theory. The Economic literature differences in terms of such concepts are examined in the light of their relationships with financial markets.
\end{abstract}

Key words: Risk, Financial risk, Financial operations, Financial markets

\footnotetext{
${ }^{1}$ Administrador, Máster in Business Administration pelo IBMEC - Brasil, Mestre em Contabilidade Internacional pela Universidad de Zaragoza - Espanha, Doutor em Finanças e Contabilidade pela Universidad de Zaragoza Espanha, Pesquisador e Professor Titular da Fundação Cultural Dr. Pedro Leopoldo. E-mail: jlp@ gold.com.br
} 


\section{Introducción}

El estudio del tema riesgo ha presentado una evolución extraordinaria en el entorno del sistema financiero internacional, llevando a académicos y profesionales de las áreas de investigación de instituciones financieras a unir conocimientos y discutir el asunto exhaustivamente.

Este proceso no ha sido ajeno a los numerosos períodos de inestabilidad y crisis financieras que han tenido lugar en los años ochenta y noventa. Las grandes crisis de esas décadas pusieron a prueba los sistemas de medición y control de los riesgos, y la necesidad de diseñar nuevos modelos y sistemas.

Desde la década de los setenta, los mercados financieros han experimentado cambios revolucionarios que han subrayado la importancia y necesidad de adecuados sistemas de control de riesgo para las entidades que en ellos operan. Las crecientes turbulencias financieras internacionales y la expansión de los instrumentos derivados, junto con las notables pérdidas sufridas por muchas instituciones (Barings con sus contratos futuros sobre el Índice Nikkei 225; Sumitomo Corp. con posiciones abiertas en el mercado de futuros sobre el cobre; Daiwa, ...) en su operativa con estos instrumentos, motivaron un intenso debate sobre la necesidad de contar con instrumentos de medición y gestión de los riesgos más precisos.

La medición del riesgo es fundamental para poder conocer con un nivel de probabilidad aceptable el valor que puede tener una cartera de valores en un momento determinado. La volatilidad de los mercados financieros, como ya fue mencionada, exige disponer de algún método de valoración del riesgo para poder gestionar correctamente cualquier operación financiera.

Según Saunders (2000, p. 178), hay por lo menos cinco motivos por los cuales la medición de riesgo de mercado es importante:

1. Información Gerencial. Suministra información a la gestión respecto de la exposición al riesgo asumida por los operadores. Esta exposición puede ser comparada al capital en riesgo.

2. Fijación de Límites. Mide el riesgo de mercado de las carteras de los operadores, permitiendo el establecimiento de límites económicamente válidos de posición por operador en cada área.

3. Destinación de Recursos. Compara resultados a riesgo de mercado en diferentes áreas de operación, lo que permite la identificación de áreas con mayor potencial de rentabilidad por unidad de riesgo, a las cuales podrían ser destinados más capital y recursos.

4. Evaluación de Desempeño. De modo semejante, permite el cálculo del cociente entre rentabilidad y riesgo de los operadores, posibilitando la implantación de un sistema racional de premiación. O sea, los operadores que obtienen las tasas de rentabilidad más elevadas pueden ser simplemente aquellos que asumieron mayores riesgos. No está claro que deban recibir mayor remuneración los operadores con menores rentabilidades y menor exposición a riesgo. 
5. Reglamentación. Ya que el BIS y la Reserva Federal de los Estados Unidos se proponen reglamentar el riesgo de mercado por medio de exigencias de capital, es importante que existan patrones de referencia en el sector privado, en el caso de que se pensara que las autoridades reguladoras están estableciendo precios excesivos para ciertos riesgos. Esos modelos generados en el sector privado pueden ser utilizados para indicar posibilidades de destinaciones eficientes de recursos como consecuencia de la reglamentación.

Una cartera (portfolio) es una combinación de inversores que permite alcanzar un determinado rendimiento. Normalmente se compone de activos financieros como títulos valores, acciones, bonos, obligaciones, etc., tanto públicos como privados, que se comportan como variables aleatorias, es decir, existe incertidumbre en cuanto su comportamiento futuro.

La teoría de carteras trata de predecir la relación entre la rentabilidad esperada y el riesgo de las carteras y de los títulos, considerados estos últimos de modo aislado. Las hipótesis en que dicha teoría se basa son las siguientes:

1. Los inversores actúan de acuerdo con las predicciones de la rentabilidad/riesgo derivadas de cada título, así como de sus correlaciones.

2. Todos los inversores se sitúan lógicamente en fronteras eficientes.

3. Cualquier inversor puede prestar los fondos que posea o pedir prestado sin límite alguno y a un único tipo de interés.

La naturaleza del problema de la selección de carteras es encontrar aquellas carteras correspondientes a las combinaciones de los activos existentes en el mercado que:

- para un determinado nivel de rendimiento tengan un riesgo mínimo; o

- para un determinado nivel de riesgo tengan rendimiento máximo.

Esas carteras componen el espacio rendimiento esperado versus riesgo, la denominada frontera eficiente.

Para Sá (1999, p. 61), el problema comporta soluciones distintas de acuerdo con las siguientes situaciones:

1. Determinación de la frontera eficiente considerándose la posibilidad de invertir solamente en títulos con riesgo.

2. Determinación de la frontera eficiente considerándose la posibilidad de que, además de invertir en títulos con riesgo, se pueda también invertir en un título de renta fija sin riesgo y, además, tomar recursos prestados para apalancar la inversión en los títulos con riesgo.

Para poder medir el riesgo que asume un inversor es necesario analizar la distribución de probabilidades subjetivas de sus rendimientos esperados. Para la cuantificación del riesgo se adjudican probabilidades a cada rendimiento posible que pueden estar basadas en frecuencias pasadas.

El primer intento de recoger la relación rentabilidad-riesgo se llevó a cabo en 1952 con el modelo de Markowitz, que dio lugar al nacimiento de la teoría clásica de carteras. Por lo tanto, partiremos nuestro estudio de la Teoría de Markowitz donde introduciremos el concepto de Reisgo. Avanzaremos con el modelo de Sharpe, donde introdiciremos la Beta, y finalizaremos con el modelo CAPM y APT. 


\section{La Medición del Riesgo de Markowitz}

Harry Markowitz ${ }^{2}$, un estudiante de doctorado norteamericano, presentó su tesis doctoral en 1952 en la Universidad de Chicago con la contribución de que la optimización de la cartera se caracteriza por una transacción entre el rendimiento esperado del título individual y la contribución de dicho título al riesgo de cartera. Su contribución inició lo que se ha llamado la Portfolio Theory o Teoría de Carteras. Con el tiempo, estas investigaciones darían lugar a una industria de gestión de inversiones basada en estos principios, traspasando la frontera académica y convirtiéndose en la manera ortodoxa de contemplar las inversiones diversificadas. Markowitz recibió el premio Nobel de Economía junto con otros discípulos suyos, Sharpe y Miller, por sus contribuciones a la teoría de carteras.

Para Pedrosa (2001, p. 21), en la década de los cincuenta y principios de la de los sesenta, la mayoría de los inversores se referían al riesgo, pero no existía una medida específica para dicho término. Dicha cuantificación no se desarrollaría hasta que Harry M. Markowitz, en su teoría de selección de carteras, derivara la tasa de rendimiento esperada de una cartera de activos y formulara una medida de riesgo esperado. Markowitz demostró que la varianza de la tasa de rendimiento era una medida de riesgo aceptable y derivó las fórmulas para calcular la varianza de una cartera de valores bajo ciertos supuestos. Además su contribución más importante, a partir de dicha fórmula, fue la demostración de la importancia de la diversificación para reducir el riesgo, es decir, la búsqueda de las proporciones en que deben combinarse los títulos óptimamente.

El planteamiento principal de la teoría de Markowitz fue la introducción de lo que se denomina comportamiento racional del inversor. Este concepto considera que todo inversor busca obtener el mayor rendimiento posible en sus inversiones, pero le preocupa también el riesgo al que se expone para obtener ese rendimiento. Igualmente, hay inversores que prefieren mayor rendimiento a expensas de un mayor riesgo, y otros que por el contrario se conforman con menos rendimiento a cambio de mayor certeza en sus ganancias.

En el modelo de Markowitz se trabajó con la primera aproximación al riesgo, que es el riesgo total, cuyo subrogante cuantitativo es la varianza (o desviación típica). A él pertenece la visión crucial de que la optimización de la cartera se caracteriza por una transacción entre el rendimiento esperado del título individual y la contribución de dicho título al riesgo de cartera.

Según López Domínguez (1998, p. 335), en el modelo desarrollado por Markowitz se parte de los siguientes supuestos fundamentales:

1. El rendimiento de cualquier título o cartera es descrito como una variable aleatoria de carácter subjetivo, cuya distribución de probabilidad para el período de referencia es conocida por el inversor.

2. El valor medio o esperanza matemática de dicha variable se acepta como medida del rendimiento o rentabilidad de la cartera.

\footnotetext{
${ }^{2}$ Markowitz, H. (1952): "Portfolio Selection", Journal of Finance, vol. 7, nº 1, March, pp. 77-91; y Markowitz, H. M. (1959): Portfolio Selection: Efficient Diversification of Investments. New Heaven: Yale University Press.
} 
3. Se acepta como medida del riesgo la dispersión, medida por la varianza o la desviación estándar, de la variable aleatoria que describe el rendimiento, ya sea de un valor individual o de una cartera.

4. La conducta del inversor le lleva a preferir aquellas carteras con un mayor rendimiento $\mathrm{y}$ un menor riesgo.

Y el riesgo de una cartera de títulos es función de tres factores:

1. Proporción, ponderación, o peso, de cada valor en la cartera.

2. La varianza o desviación estándar de cada valor.

3. La covarianza o coeficiente de correlación entre cada par de valores.

Para Pascale (1999, p. 213-214) pueden distinguirse en el enfoque de Markowitz cuatro etapas para la constitución y selección de carteras óptimas:

1. La primera etapa consiste en determinar a partir de qué activos se va a trabajar para elaborar la frontera de eficiencia; esto es, el conjunto de carteras óptimas. En ella, habitualmente, participan el inversor y algún asesor en inversiones.

2. La segunda etapa tiene que ver con el análisis de los activos elegidos en la anterior. Este análisis deberá terminar con la determinación de los rendimientos esperados, varianzas y covarianzas de todos los activos que están en consideración. En esta fase, la responsabilidad básica recaerá sobre el analista de activos.

3. La tercera etapa es la determinación del conjunto de carteras eficientes. A partir de los rendimientos esperados, las varianzas y las covarianzas de los activos involucrados, que se calcularon en el paso anterior, se determina la composición de activos, asimismo el riesgo y el rendimiento de las carteras eficientes que componen el conjunto eficiente. Este concepto conforma la frontera de eficiencia en forma de curva cóncava creciente, que vincula el riesgo (medido por la varianza) con los rendimientos esperados. Esta fase, habitualmente, está en manos de un gestor de carteras.

4. La cuarta etapa es la elección, por parte del inversor, de la cartera óptima en términos de su apreciación subjetiva sobre el riesgo. En esta etapa, la participación del inversor es decisiva. Frecuentemente, éste es aconsejado por un asesor en inversiones.

El modelo de Markowitz está elaborado a partir de activos arriesgados. No existe en él un activo libre de riesgo, esto es, que tenga rendimientos conocidos con certidumbre. El modelo no considera la posibilidad de construir una frontera de eficiencia en presencia de activos riesgosos y de un activo libre de riesgo.

La frontera de eficiencia de Markowitz, construida con activos arriesgados, se conforma sobre la base de los rendimientos esperados y las varianzas, y el óptimo se verifica en la tangencia de una curva de indiferencia del inversor con la frontera. 
Grafico 2.1 - Línea del Mercado de Capitales

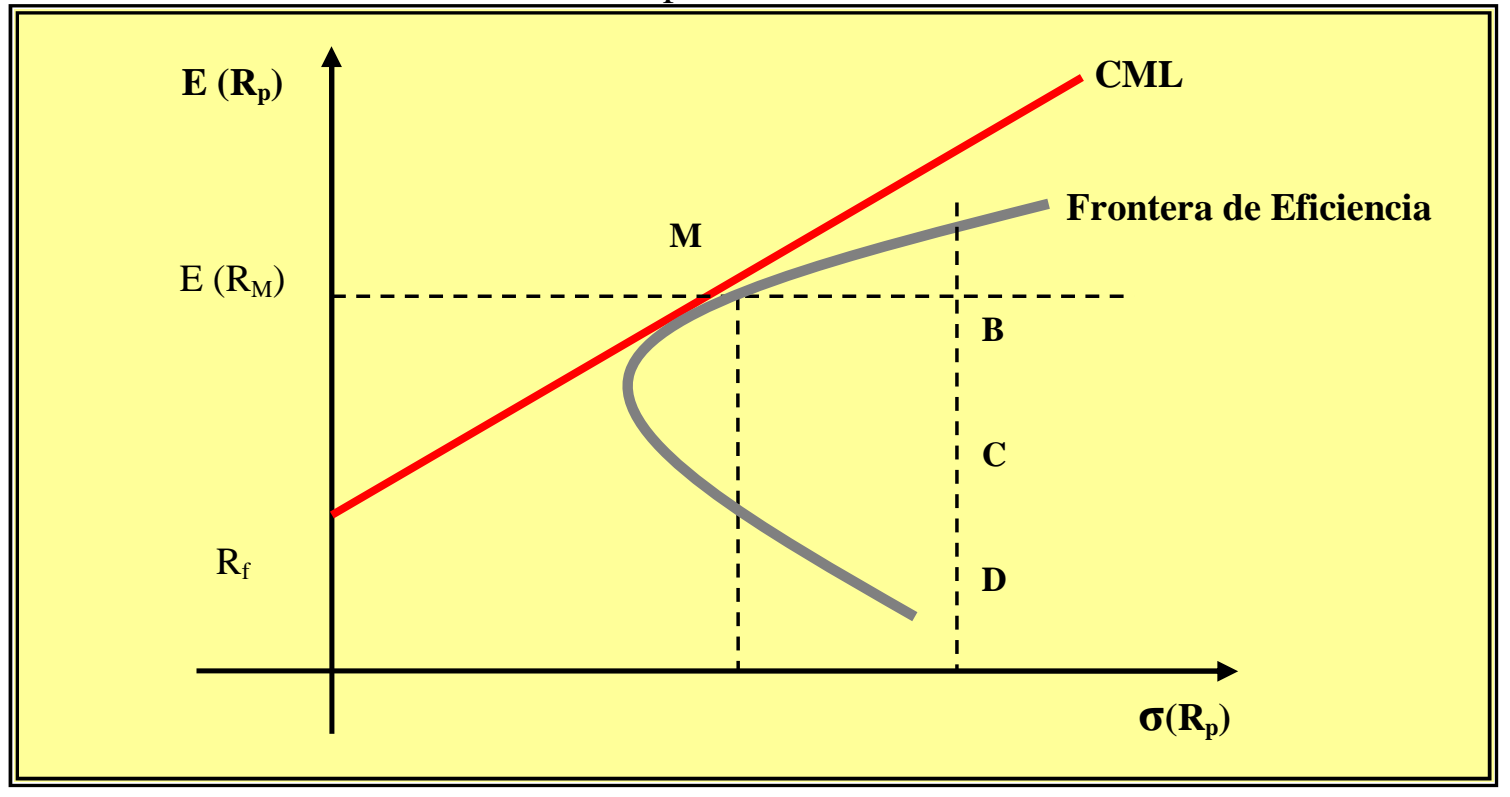

La Línea de Mercado de Capitales o Capital Market Line (CML) describe la relación riesgo/rendimiento para carteras eficientes; esto es, para carteras que resultan de una mezcla de cartera de mercado y un activo libre de riesgo.

Otros resultados que Markowitz encuentra aplicados a la medición de riesgo son:

- El riesgo de una cartera de inversión tiene dos componentes: riesgo diversificable y riesgo no diversificable.

- Cuando la cartera de inversión está conformada con $N$ activos, con igual participación en la misma, cada uno con riesgo $\sigma$ y rendimientos no correlacionados, el riesgo de la cartera será igual a $\sigma$ dividido por la raíz cuadrada de $N$. Es decir, conforme aumenta $N$, menor es el riesgo de la cartera.

El abordaje de Markowitz para la optimización de carteras, mientras sea intuitivamente atrayente, sufre dos gran problemas. Un es la necesidad de un gran número de datos de entrada, una vez que las covariancias entre pares de activos son imputs obligatorios para estimar las variancias de carteras.

Mientras que tal hecho posa ser administrable para pequeñas cantidades de activos, plantease más difícil cuando considerase todo un universo de acciones o todas las inversiones.

El según problema es que la abordaje de Markowitz ignora una elección de activos muy importante disponible para la mayoría de los inversores, o sea, la elección de investir su dinero sin cualquier risco en títulos do gobierno libres de retraso, al elaboraren carteras optimizadas. 


\section{La Medición del Riesgo de Sharpe: El CAPM}

El modelo de valoración de activos, conocido por sus iniciales en inglés CAPM (Capital Asset Pricing Model), nació en los años sesenta de la mano de Sharpe (1964 y 1991), Lintner (1965), Treynor (1965) y Mossin (1969). Posteriormente otros autores hicieron aportaciones importantes, como es el caso de Black (1972).

El modelo ideado por Markowitz no había sido superado en su concepto matemático. El problema radicaba en el gran número de ecuaciones que deben desarrollarse cuando el número de valores en cartera es alto.

Sharpe, en 1963, simplificó el procedimiento matemático creando el llamado modelo de mercado, que ha sustituido con éxito al de Markowitz. Él centra su estudio en el binomio rentabilidad-riesgo y establece que la rentabilidad de una cartera viene definida por la medida ponderada de las rentabilidades esperadas de los valores que la componen:

- Un componente individual que refleja la parte de la variación de la rentabilidad del título que es independiente de la rentabilidad del mercado, y que depende únicamente de factores internos de cada empresa o industria. La variación del componente individual, denominado riesgo no sistemático, puede ser eliminada mediante una adecuada diversificación de la cartera.

- Un componente sistemático, donde un coeficiente beta refleja la variación de la rentabilidad de un título con respecto a la rentabilidad del mercado. Por tanto, beta será el riesgo sistemático, y que no puede eliminarse por medio de la diversificación.

Sharpe (1964) y Linter (1965) ampliaron el modelo inicial a un modelo de precios (CAPM), que determina el precio de equilibrio de todos los títulos del mercado. El CAPM surgió como respuesta a la siguiente pregunta: ¿Qué cartera de acciones y renta fija debe formar un inversor que tiene aversión al riesgo? Por aversión al riesgo se entiende que a igualdad de rentabilidad esperada, un inversor siempre preferirá la cartera con menor riesgo.

Según Pascale (1999, p. 214-215), el CAPM se asienta para su desarrollo en un conjunto de supuestos que son:

1. Los inversores son diversificadores eficientes de inversiones en el sentido dado por Markowitz. Esto es, buscan formar carteras eficientes. Los supuestos del mismo se asientan en que los inversores son aversos al riesgo, el cual miden a través de la desviación estándar de los rendimientos de la cartera.

Este supuesto hace posible trabajar, luego, sobre la base de riesgos determinados por beta.

2. Todas las inversiones tienen, para su planificación, el mismo período; por ejemplo, un trimestre, un año, etc. El CAPM, al igual que el modelo de cartera, es uniperiódico.

3. Los inversores tienen expectativas homogéneas; por tanto, visualizan idénticas funciones de probabilidad para rendimientos futuros.

4. Existe un mercado de capitales perfecto, lo que implica:

- Todos los activos son perfectamente divisibles y comerciables

- No hay costos de transacciones ni de información

- No existen impuestos

- Cada comprador o vendedor tiene efectos prácticamente insignificantes sobre el mercado 
- Existe cualquier cantidad de dinero para prestar o pedir prestada a un mismo tipo de interés para los inversores

5. Existencia de una tasa libre de riesgo e ilimitadas probabilidades de pedir prestado y prestar a una tasa.

6. Inexistencia de inflación.

Para Fernández (2001, p. 386), las consecuencias fundamentales del CAPM, son:

1. Cualquier combinación de bonos sin riesgo y de la cartera del mercado domina a cualquier otra combinación de acciones y bonos.

2. Todos los inversores tendrán una cartera compuesta en parte por renta fija sin riesgo y en parte por la cartera del mercado. Las proporciones serán distintas según su función de utilidad.

3. La cartera del mercado se compone de todos los activos que existen, y la cantidad de cada uno es proporcional a su valor de mercado.

Para Gómez-Bezares (1999, p. 6), la afirmación fundamental del CAPM es que la rentabilidad esperada de un activo ha de ser función lineal de su riesgo sistemático, medido por beta. Partiendo de que los individuos son enemigos del riesgo, y que el riesgo relevante es el sistemático, que se mide con beta (el resto del riesgo se eliminará por diversificación), es fácil intuir que la rentabilidad esperada en cualquier inversión deberá ser mayor cuanto mayor sea beta.

La discusión del modelo puede ser establecida en tres partes. La primera definiendo y describiendo el coeficiente beta, que es una medida de riesgo sistemático, la segunda presentando una ecuación del modelo y la tercera describiendo gráficamente la relación riesgo rentabilidad.

Gómez-Bezares et al. (1996, p. 73) nos dicen que, según el modelo, el único riesgo relevante, el único que debe ser retribuido, es el que se denomina "riesgo sistemático", y propone una medida del mismo, la beta.

El coeficiente beta es una medida del grado de relación de la rentabilidad de un título con la del mercado. Dicho coeficiente es un índice del grado de movimiento de la rentabilidad de un activo en respuesta a un cambio en la rentabilidad del mercado. El coeficiente beta se define como en la expresión [2.01].

$$
\beta_{\mathrm{i}}=\frac{\operatorname{COV}\left(\mathrm{R}_{\mathrm{i}}, \mathrm{R}^{*}\right)}{\operatorname{VAR}\left(\mathrm{R}^{*}\right)}
$$

Es decir, como cociente entre la covarianza de la rentabilidad del título con el mercado y la varianza de rentabilidad de este último. Esta medida puede obtenerse en el llamado "Modelo de Mercado", que propone un ajuste de regresión entre la rentabilidad del título y la correspondiente al mercado, en el que la pendiente del ajuste coincidiría con la mencionada beta. 


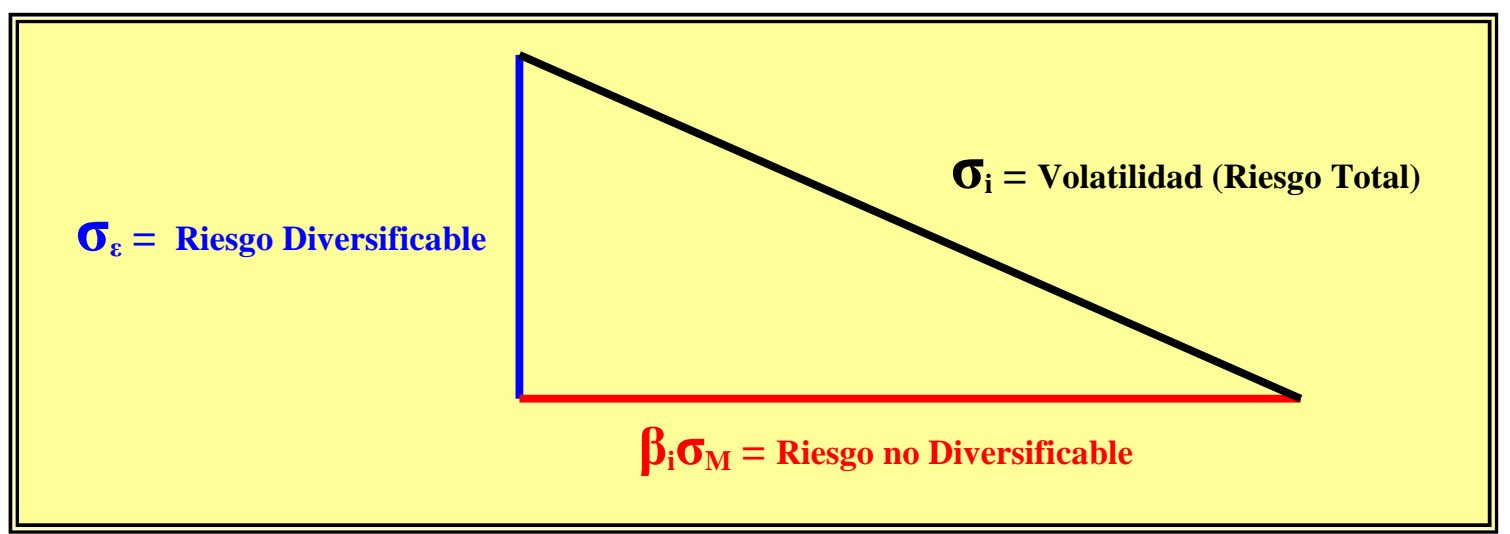

Figura 2.1 - Relación entre Beta y Volatilidad

El CAPM propone que la rentabilidad esperada de un título es función de su riesgo sistemático, como en la expresión [2.02].

$$
\mathrm{E}\left(\mathrm{R}_{\mathrm{i}}\right)=\mathrm{R}_{0}+\left[\mathrm{E}\left(\mathrm{R}^{*}\right)-\mathrm{R}_{0}\right] \times \beta_{\mathrm{i}}
$$

Donde:

$\mathrm{E}\left(\mathrm{R}_{\mathrm{i}}\right)=$ Rentabilidad esperada del título $\mathrm{i}$.

$\mathrm{R}_{0} \quad=$ Rentabilidad del título sin riesgo (renta fija).

$\mathrm{E}\left(\mathrm{R}^{*}\right)=$ Rentabilidad esperada de la cartera de mercado (teóricamente compuesta por todos los activos que aportan valor a la economía).

$\beta_{\mathrm{i}} \quad=$ Beta del título $\mathrm{i}$. Es una medida de su riesgo sistemático.

Cuando se representa gráficamente la relación entre la beta de una cartera y el rendimiento de dicha cartera, se obtiene la llamada Línea de Mercado de Títulos o Security Market Line (SML).

Grafico 2.2 - Línea del Mercado de Títulos 


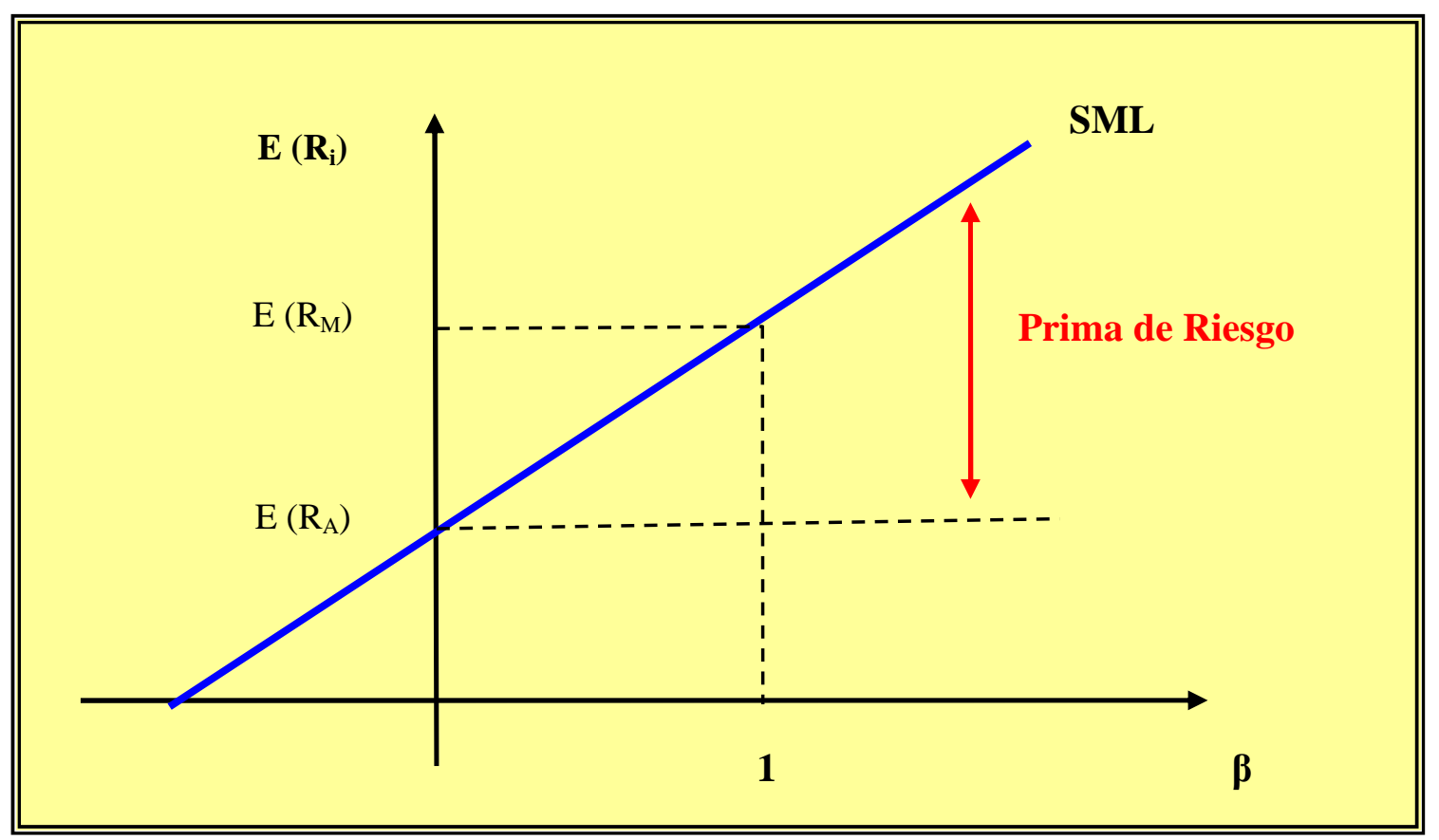

Hay tres aspectos importantes asociados al Gráfico 2.2.

\section{Una beta igual a cero.}

El rendimiento esperado de un título con beta igual a cero es obtenido por la tasa libre de riesgo. Como un título con beta nulo no posee riesgo relevante, su rendimiento esperado sólo puede ser igual al del activo.

\section{Una beta igual a un.}

Indica que la beta media de todos los títulos, cuando se pondera por la proporción del valor de mercado de cada título en relación al de la cartera de mercado, es igual a 1. Como la cartera de mercado se forma ponderándose cada título por su valor de mercado, el beta de la cartera de mercado es igual a 1. Basándose en el hecho de que todos los títulos con idéntica beta tienen el mismo rendimiento esperado, el rendimiento esperado para cualquier título con beta igual a 1 debe ser el rendimiento esperado de la cartera de mercado.

\section{Linealidad.}

La intuición de que la curva debe ser ascendente es muy clara. Como la beta es la medida apropiada de riesgo, los títulos con betas elevados deben tener un rendimiento esperado superior al de títulos con betas reducidos. Entretanto, el Gráfico 2.02 presenta algo más específico que una simple curva ascendente: dice que la relación entre rendimiento esperado y beta es dada por una línea recta.

Según Gómez-Bezares et al. (1996, p. 74), todos los títulos se situarían en la SML. Pero, en la medida en que el CAPM no cumpliera exactamente en la realidad, los títulos aparecerían alrededor de la recta propuesta. Ello podría deberse a multitud de motivos, entre ellos el hecho de que la verdadera cartera de mercado es imposible de conocer, y trabajamos siempre con estimaciones de la misma. Pero también podemos suponer que el mercado está equivocado, y no actúa eficientemente. Esto provocaría la existencia de títulos que quedan por encima de la línea (que, al rendir más de lo que cabría exigírseles en función de su riesgo sistemático, estarían 
infravalorados), y títulos que se sitúan por debajo de la línea (que estarían sobrevalorados). Si aceptamos la lógica del modelo, y suponemos que esta situación se va a mantener en el futuro, deberíamos comprar los primeros y deshacernos de los segundos, constituyendo el CAPM una herramienta para la toma de decisiones en bolsa.

Para Weston y Copeland (1995, p. 462), la Línea del Mercado de Capitales y la Línea del Mercado de Títulos representan sólo diferentes panoramas del mismo equilibrio de mercado. La CML se puede usar para determinar el rendimiento requerido tan sólo para aquellas carteras eficientes que se encuentran perfectamente correlacionadas con la cartera de mercado porque caen sobre la CML, pero la SML puede usarse para explicar la tasa requerida de rendimiento sobre todos los valores, indistintamente de que sean o no eficientes. La SML proporciona una relación única entre el riesgo no diversificable (medido por $\beta$ ) y el rendimiento esperado. Por tanto, si puede medir en forma exacta el beta de un valor, puede estimarse su tasa de rendimiento en equilibrio ajustada por el riesgo. 


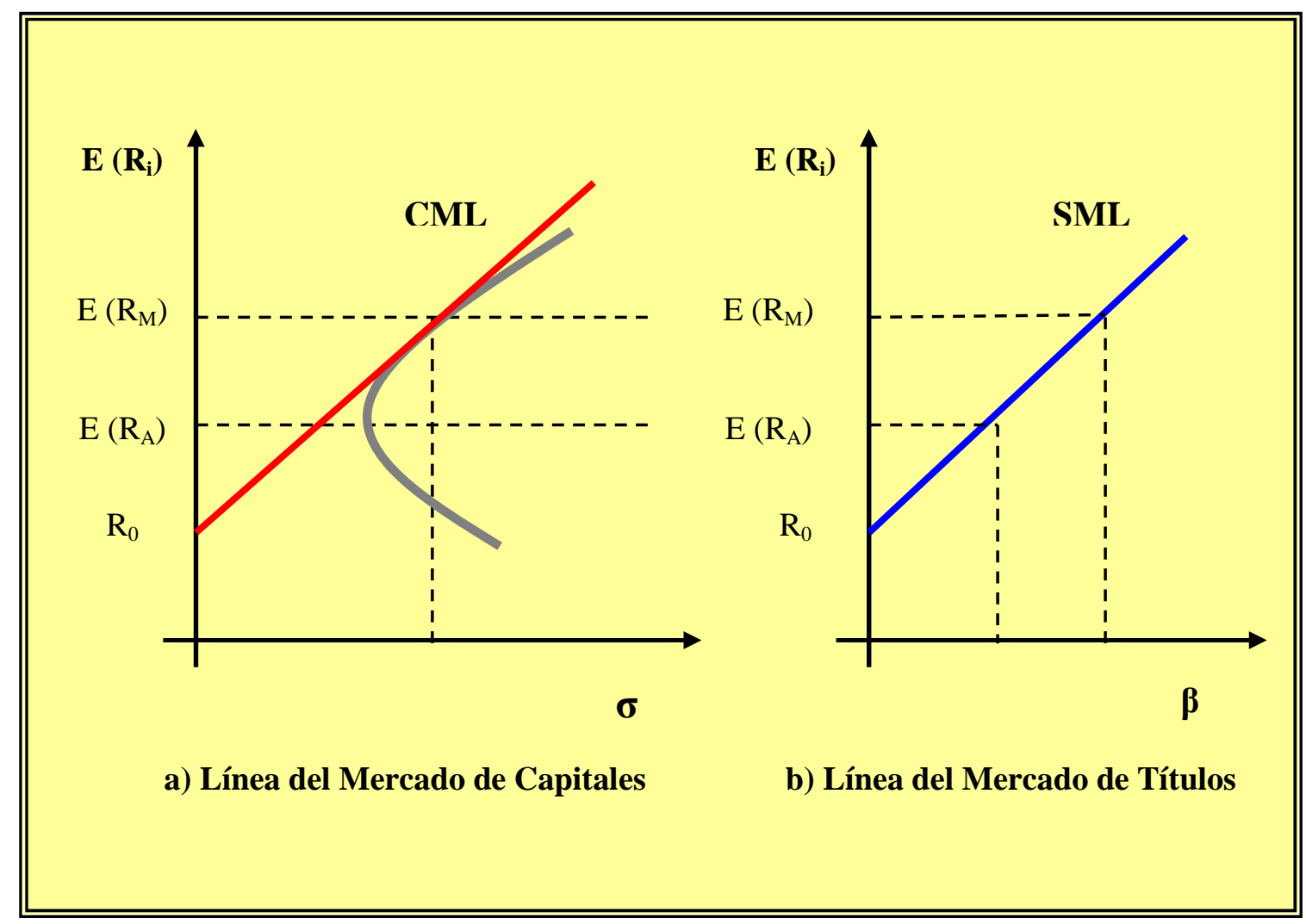

Figura 2.2 - Comparación de las Líneas de Mercado de Capitales con la del Mercado de Títulos

Fuente: Weston y Copeland (1995, p. 483)

Para Ross et al. (1995, p. 226), las dos líneas son muy diferentes. La CML representa el conjunto de carteras eficientes formadas tanto por activos con riesgo cuanto por activo sin riesgo. Cada punto de esta línea representa una cartera entera. Sus ejes miden el rendimiento esperado de una cartera y la desviación estándar del rendimiento de una cartera. Los títulos individuales no se sitúan sobre la línea.

La SML relaciona rendimiento esperado y beta. Por tanto los gráficos de la CML y SML difieren, por lo menos, en dos sentidos: la beta aparece en el eje horizontal de la SML, mientras que en la CML la desviación estándar es medida en ese eje; la SML se refiere tanto a todos los títulos individuales como a todas las carteras posibles, al tiempo que la CML vale apenas para carteras eficientes.

El CAPM es extremamente atrayente en un nivel intelectual: ello es lógico y racional, y una vez que alguien trabaja y entiende la teoría, su reacción es, de forma general, acéptalo sin cuestionamiento. Entretanto, las dudas empiezan a surgir cuando se piensa respecto a los presupuestos sobre los cuales el modelo está basado, y esas dudas son tanto reforzadas cuanto reducidas por los tests empíricos ${ }^{3}$. Douglas (1969) fue el primero en tener dudas sobre el contenido empírico del modelo.

\footnotetext{
${ }^{3}$ Una lista parcial de las pruebas empíricas del CAPM incluye a Douglas (1969), Bume y Friend (1970, 1973), Blake, Jesen y Scholes (1972), Millar y Scholes (1972, 1982), Blume y Husick (1973), Fama y Macbeth (1973), Basu (1971), Reinganum (1981), Litzenberger y Ramaswamy (1979, 1982), Banz (1981), Stambaugh (1982), Gibbons (1982) y Keim (1983).
} 
Douglas descubrió evidencias condenatorias en dos instancias: primero el riesgo nosistemático parecía explicar las rentabilidades medias y, segundo, la línea estimada de mercado de títulos era por demás superficial, es decir, su intercepto era mayor que de área libre de riesgo, lo que significa que las acciones defensivas $(\beta<1)$ tenían la tendencia de tener alfas positivas, mientras que las agresivas $(\beta>1)$ tenían la tendencia de tener alfas negativos.

\section{La Medición del Riesgo de Ross: El APT}

Derivada por primera vez por Ross ${ }^{4}$, en 1976, aunque el supuesto de ausencia de arbitraje, eje fundamental del modelo, ya había sido utilizado por Black y Scholes ${ }^{5}$ en 1973. La teoría de la Fijación de Precios de Arbitraje o Arbitrage Princing Theory (APT) parte del hecho de que la tasa de rendimiento sobre cualquier valor es una función lineal del movimiento de un conjunto de factores fundamentales.

Según López Domínguez (1998, p. 343), en este método, el riesgo sistemático es el factor explicativo fundamental del comportamiento de la rentabilidad de los activos financieros, pero medido a través de $\mathrm{n}$ factores explicativos a priori. Se trata de un modelo más general que el CAPM y que no se basa en la hipótesis de eficiencia del mercado, sino que parte de que en un mercado en equilibrio no puede haber oportunidades de inversión sin explotar, es decir, que ningún inversor mediante el arbitraje, conseguirá rendimientos superiores a los que obtenía en la anterior composición de su cartera.

Por tanto, la rentabilidad dependerá de n factores, como presenta la expresión [2.03].

$$
E(R)=R+b_{1} \cdot f_{1}+b_{2} \cdot f_{2}+\ldots b_{n} \cdot f_{n}
$$

Donde:

$\mathrm{b}_{\mathrm{i}}=$ Sensibilidad para el factor $\mathrm{i}$.

$\mathrm{f}_{\mathrm{i}} \quad=$ Prima de riesgo para el factor $\mathrm{i}$.

$\mathrm{R}=$ Rentabilidad del activo libre de riesgo.

El modelo puede ser visto como una generalización del CAPM, porque suministra el rendimiento esperado de un activo por encima de la tasa libre de riesgo, como resultado de una ponderación entre los precios del riesgo de cada uno de los factores de rendimiento básicos del mercado.

\footnotetext{
${ }^{4}$ Ross, S. (1976): “The Arbitrage Theory of Capital Asset Pricing”, Journal of Economic Theory, December, pp. 341-361.

5 BLACK, E. y SCHOLES, M. (1973): "The Pricing of Options and Corporate Liabilities", Journal of Political Economy, 81, pp. 637-659.
} 
Cuadro 2. 1 - Variables Macroeconómicas Relacionadas con la APT

\begin{tabular}{|ll||}
\hline 1. Producción industrial (o cartera de mercado). \\
2. Cambios de la prima de riesgo de incumplimiento (medidos por \\
las diferencias de los rendimientos prometidos al vencimiento \\
sobre bonos corporativos AAA versus Baa). \\
3. Distorsiones de la curva de rendimiento (medidas por las \\
diferencias entre los rendimientos prometidos al vencimiento \\
sobre bonos del gobierno a corto y a largo plazos). \\
4. Inflación no anticipada. \\
5. Cambios de la tasa real (medidos por la tasa de los Certificados \\
de la Tesorería menos el índice de precios al consumidor).
\end{tabular}

Fuente: Weston y Copeland (1995, p. 483)

Según Brigham et al. (2001, p. 231), la principal ventaja teórica del APT es que permite que varios factores económicos influencien los rendimientos de las acciones individuales, mientras el CAPM asume que el impacto de todos los factores, excepto aquellos exclusivos de la empresa, puede ser capturado en una única medida: la volatilidad de la acción con respecto a la cartera de mercado. Además de eso, el APT requiere menos presupuestos que el CAPM y, por tanto, es una teoría más general. Finalmente, el APT no presupone que todos los inversores mantengan la cartera de mercado, una exigencia del CAPM que claramente no es encontrada en la práctica.

Entretanto, el APT encuentra varias barreras en su implantación. La más severa es que el APT no identifica los factores relevantes. Así, el APT no presenta cuáles son los factores que influencian los rendimientos y tampoco indica cuántos factores deberían aparecer en el modelo.

\section{Cuadro 2.02}

\section{Comparación entre el CAPM y el APT}

\section{CAPM}

El CAPM declara que, en media, apenas un beta del activo es recompensado. O sea, el rendimiento sobre cualquier activo es la tasa sin riesgo más el beta, multiplicado por la prima del riesgo de mercado. Esto puede ser expresado como:

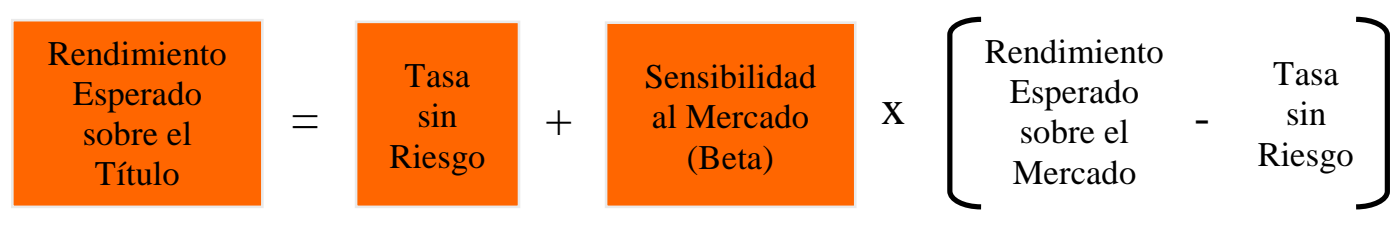

\section{APT}

El APT reconoce que puede haber varios factores difusos. Si los activos fueran afectados de forma diferente por esos factores, entonces la consideración de cada uno de los factores será vital. El rendimiento esperado sobre el activo es la tasa libre de riesgo más la sensibilidad del activo a cada factor, multiplicada por la prima de riesgo asociada al factor. A modo de ejemplo, con dos factores puede ser expresado como: 


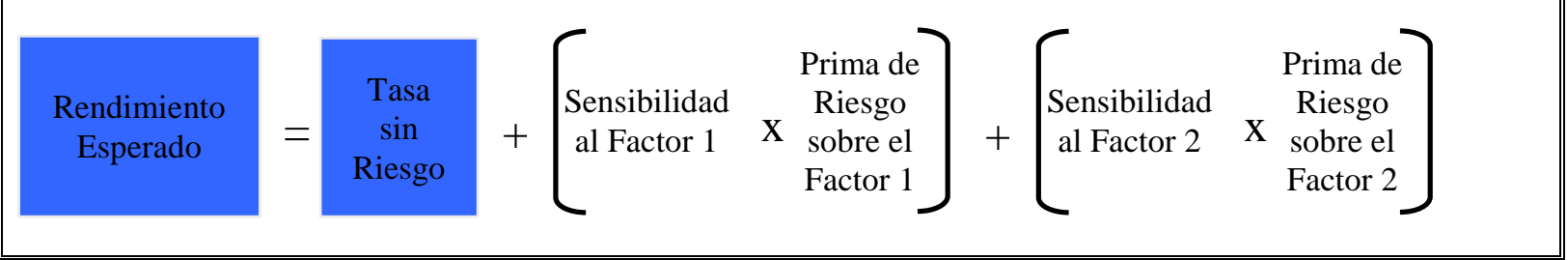

Gráficamente, el concepto del CAPM se ilustra en el Grafico 2.03, donde aparece representado por una línea (SML), entorno a la cual todos los activos deben se ajustarse El concepto de CAPM se expresa en el Gráfico 2.04, en este caso se representa por un plano sobre que todos los activos deben figurar.

Gráfico 2. 3 - Línea del Mercado de Capitales del CAPM

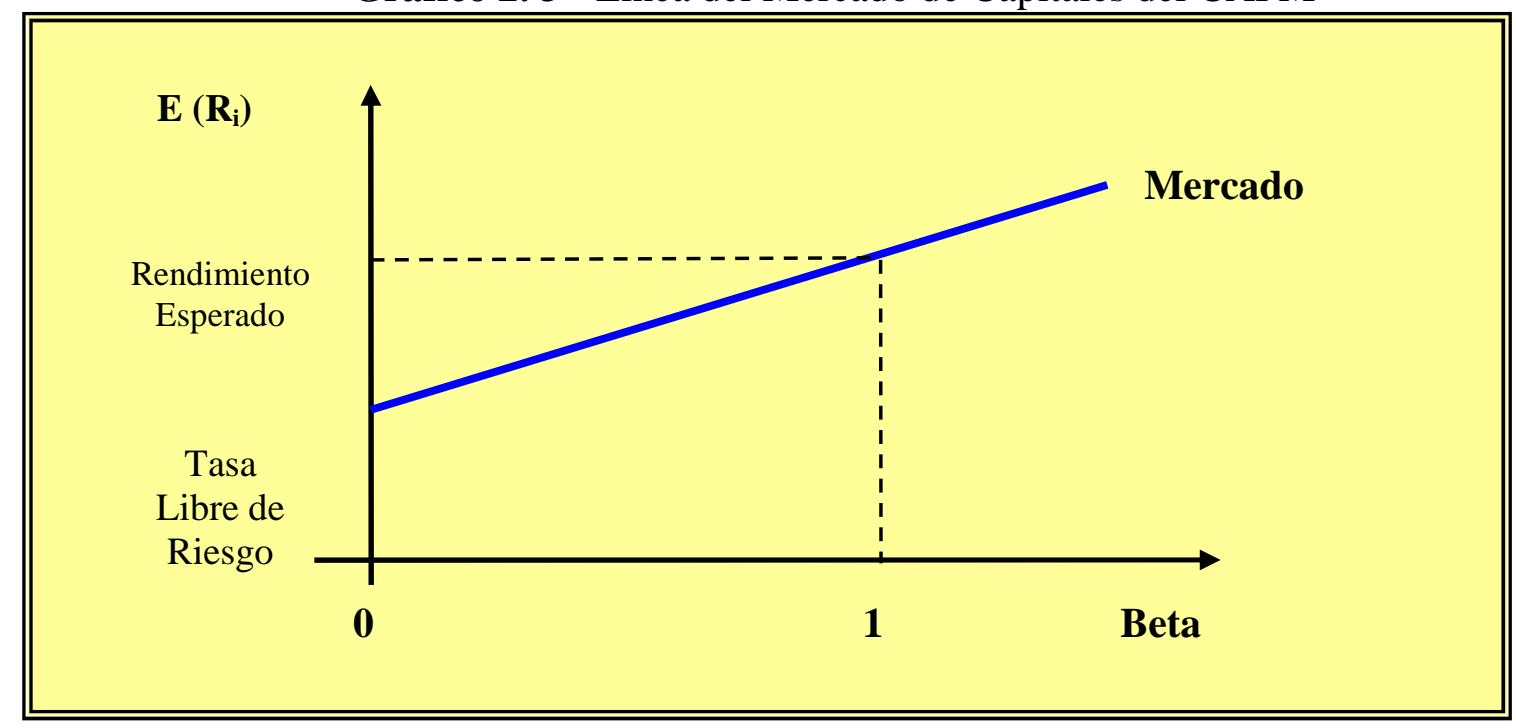

Gráfico 2.04 - El APT

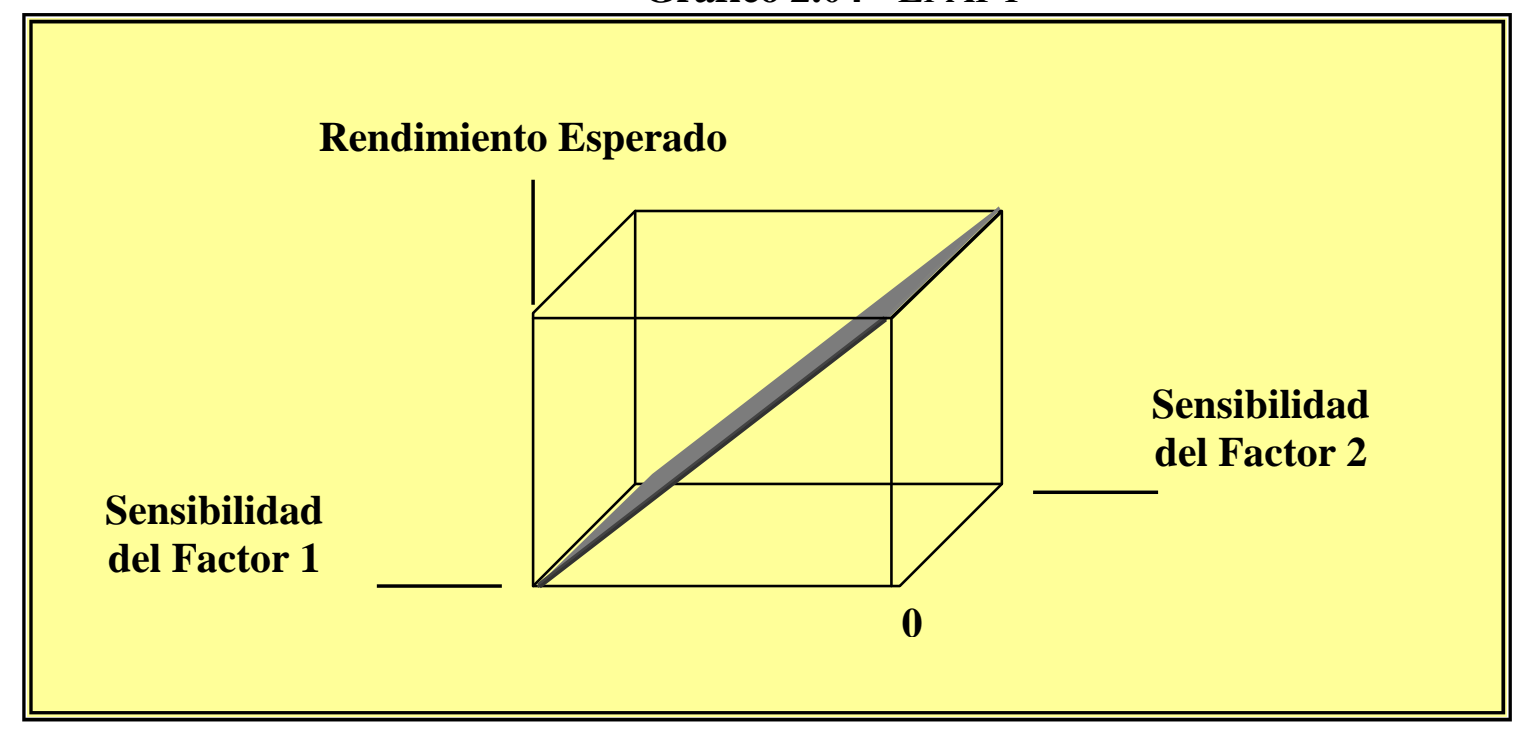




\section{Consideraciones Finales}

Los conceptos descritos en este capítulo son una introducción a la interpretación de la medición del riesgo de mercado en la Teoría de Cartera. Cuando se trabaja con modelos econométricos es fundamental el conocimiento de las premisas y de sus puntos fuertes y débiles.

Pasando por Markowitz (1952) que definió el riesgo en la área financiera como siendo la variancia o desviación en relación a una media, excluyendo la ocurrencia de una tasa libre de riesgo. Sharpe (1964) que presento los conceptos del CAPM (Capital Asset Pricing Model), donde el riesgo de un activo para un inversor es el riesgo que este activo acrecienta a la cartera de mercado. Llegamos a Ross (1976) con el ATP - construyendo a partir de los principios de noarbitraje, los retornos de los activos son representados por medio de una combinación linear de los retornos líquidos de varios factores, riscos específicos para los diferentes activos e innumeros factores.

\begin{tabular}{|c|c|c|c|}
\hline Cuadro Comparativo & Markowitz & CAPM & APT \\
\hline Contribuciones & $\begin{array}{c}\text { Fue el pionero en } \\
\text { la diversificación } \\
\text { de títulos para } \\
\text { obtener mayor } \\
\text { retorno con } \\
\text { menor riesgo. }\end{array}$ & $\begin{array}{c}\text { Innovo utilizando } \\
\text { retorno libre de } \\
\text { riesgo en su } \\
\text { modelo. }\end{array}$ & $\begin{array}{c}\text { Por el hecho de } \\
\text { considerar varios } \\
\text { factores, crió la } \\
\text { posibilidad de medir } \\
\text { retornos esperados mas } \\
\text { precisamente que el } \\
\text { CAPM. }\end{array}$ \\
\hline $\begin{array}{c}\text { Diversificación de cartera; } \\
\text { baja covariancia }(\beta)\end{array}$ & Sin & Sin & Sin \\
\hline Tasa Libre de Riesgo & $\mathrm{X}$ & Sin & Sin \\
\hline Factor & 1 & 1 & $\begin{array}{c}\text { Relativo y } \\
\text { Uniforme }\end{array}$ \\
\hline Riesgo & $\begin{array}{c}\text { Relativo y } \\
\text { Uniforme }\end{array}$ & $\begin{array}{c}\text { Específico para cada } \\
\text { activo }\end{array}$ \\
\hline
\end{tabular}

Los modelos estudiados son alternativas para perfeccionar las aproximaciones de los valores futuros. La evolución de los modelos para medición del riesgo de mercado ha sido acelerada en todo el mundo después de las crisis internacionales. Las inversiones en el tema de cuantificación de riesgo son de billones de dólares. Además, eventos como el Comité de Basilea y de la concesión de algunos premios Nobel a investigadores en esa área ${ }^{6}$ están contribuyendo cada día a la mejora de las metodologías de cálculo de riesgo.

Para la determinación de la variabilidad de los rendimientos medios de una cartera es necesario conocer los riesgos de cada activo y la correlación entre cada par de rendimientos. El riesgo medio de una cartera puede ser menor que el de cualquiera de los activos que la componen, pero en general siempre será menor que la media ponderada de los riesgos de los activos que la componen.

\footnotetext{
${ }^{6}$ La investigación de Harry Markowitz, Merton Millar y Willian Sharpe relacionada con selección óptima de carteras es premiada en 1990, mientras que los métodos de valorización de derivados de Robert Merton y Myron Scholes les hace merecedores al premio Nobel de 1997.
} 


\section{Bibliografía}

BARQUERO CABRERO, J. D. y HUERTAS COLOMINA, F. J. (1998): Manual de Banca, Finanzas y Seguros. $2^{\text {a }}$ ed. Barcelona: Gestión 2000

BERNSTEIN, R. y TEW, B. (1991): "The Equity "Yield Curve"”, The Journal Portfolio Management, Fall, pp. 35-39.

BERTRAN JORDANA, J. (1994): "Control y Gestión de los Riesgos Financieros", Alta Gestión, $n^{\circ} 173$, pp. 65-72.

BLACK, E. y SCHOLES, M. (1973): "The Pricing of Options and Corporate Liabilities", Journal of Political Economy, 81, pp. 637-659.

BLACK, F. (1972): "Capital Market Equilibrium with Restricting Borrowing”, Journal of Business, 45, July, pp. 444-454.

BRIGHAM, E. F.; GAPENSKI, L. C. y EHRHARDT, M. C. (2001): Financial Management: Theory and Practice, 9 ${ }^{a}$ ed., New York, Dryden Press, 1999 (tr. brasileña de José Carlos Guimarães Alcântara, "Administração Financeira”, Atlas São Paulo 2001).

CASILDA BÉJAR, R.; LAMOTHE FERNÁNDEZ, P. y MONJAS BARROSO, M. (1997): La Banca y los Mercados Financieros. Madrid: Alianza.

FERNÁNDEZ, P. (2001): Valoración de Empresas: Cómo Medir y Gestionar la Creación de Valor. Barcelona: Gestión 2000.

FERRUZ AgUDO, L.; PORTILlO TARRAgONA, M. P. y SARTO MARZAL, J. L. (2001): Dirección Financiera del Riesgo de Interés. Madrid: Pirámide.

FISHER, L. (1966): “An Algorithm for Finding Exact Rates of Return", Journal of Business, 1, pp. 111-118.

GÓMEZ-BEZARES, F. (1999): "El CAPM: Un Modelo Útil y Polémico", Análisis Financiero, ${ }^{\circ} 78$, pp. 6-18.

GÓMEZ-BEZARES, F.; MADARIAGA, J. A. y SANTIBÁÑEZ, J. (1996): "Modelos de Valoración y Eficiencia: ¿Bate el CAPM al Mercado?”, Análisis Financiero, nº 68, pp. 72-81.

HICKS, J. R. (1976): Value and Capital, An Inquiry into Some Fundamental Principles of Economic Theory. $1^{a}$ ed., Oxford, The Cladenron Press, 1939 (tr. española de Javier Márquez, "Valor y Capital", en Fondo de Cultura Económica, Bogóta 1976, 4" reimp.).

HOPEWELL, M. y KAUFMAN, G. (1973): "Bond Price Volatility and Years to Maturity", American Economic Review, September, pp. 149-753. 
JORION, P. (1999): Value at Risk. $1^{\text {a }}$ ed., New York, McGraw-Hill Companies, 1997 (tr. española de Juan Gonzáles Herrera y Jaime Días Tinoco, "Valor en Riesgo", Limusa, México 1999).

LINTER, J. (1965): “Security Prices, Risk and Maximal gains from Diversification", Journal of Finance, December, pp. 587-616.

MACAULAY, F. R. (1938): Some Theoretical Problems Suggested by the Movements of Interest Rates, Bonds, Yields and Stock Prices in the United Status since 1856, New York: National Bureau of Economic Research.

MARKOWITZ, H. (1952): "Portfolio Selection", Journal of Finance, vol. 7, n 1, March, pp. 77-91.

MARKOWITZ, H. M. (1959): Portfolio Selection: Efficient Diversification of Investments. New York: John Wiley \& Sons.

MORALES MARTINOL, C. (1998): El Control de los Riesgos de Mercado por medio de los Productos Derivados. Publicado en Manual de Banca, Finanzas y Seguros. Barcelona: Gestión 2000, pp. 297-326.

MOSSIN, J. (1969): "Security Pricing and investment criteria in Competitive Markets", American Economic Review, 59, December, pp. 749-756.

PARRO CUESTA, E. (1994): “los Riesgos de Inversión y la Gestión de Carteras en las Entidades Aseguradoras", Análisis Financiero, nº 62, pp. 94-107.

PASCALE, R. (1999): Decisiones Financieras. $3^{\mathrm{a}}$ ed. Buenos Aires: Macchi.

PEDROSA, M. (2001): Apuntes de los mercados Financieros Internacionales. Madrid: Editorial AC.

PRIETO PÉREZ, E. (1993): "los Riesgos de Inversión y la Gestión de Carteras en las Entidades Aseguradoras", Análisis Financiero, n 59, pp. 94-100.

RAMÓN ARAGONÉS, J. y BLANCO, C. (2000): Valor en Riesgo: Aplicación a la Gestión Empresarial. Madrid: Pirámide.

RAYO CANTON, S. (1994): "Gestión del Riesgo de Interés en Bancos y Cajas de Ahorros. Una Análisis desde el Margen Financiero", Actualidad Financiera, no 16-18, pp. 231-273.

REDINGTON, F. M. (1952): "Review of the Principle of Life Office Valuations", Journal of the Institute of Actuaries, 18, pp. 286-340.

ROSS, S. (1976): “The Arbitrage Theory of Capital Asset Pricing”, Journal of Economic Theory, December, vol. 13, pp. 341-360. 
ROSS, S. A.; WESTERFIELD, R. W. Y JAFFE, J. F. (1995): Corporate Finance, $3^{\text {a }}$ ed., New York, McGraw-Hill Companies, 1993 (tr. Brasileña de Antonio Zoratto Sanvicente, “Administração Financeira: Corporate Finance”, Atlas São Paulo 1995).

SÁ, G. T. (1999): Administração de Investimentos: Teoria de Carteiras e Gerenciamento do Risco. Rio de Janeiro: Qualitymark.

SAMUELSON, P. (1945): "The Effect of Interest Rates Increases on the Banking System", American Economic Review, pp. 16-27.

SÁNCHEZ CERÓN, C. (2001): Valor en Riesgo y Otras Aproximaciones. México: SEI Investments de México.

SHARPE, W. F. (1964): "Capital Asset Prices: A Theory of Market Equilibrium under Conditions of Risk", Journal of Finance, September, pp. 425-442.

(1991): "Capital Asset Prices with and without Negative Holdings", Journal of Finance, June, pp. 489-509.

SMITHSON, C. (1998): Managing Financial Risk - A Guide to Derivative Products, Financial Engineering, and Value Maximization. $3^{\mathrm{a}}$ ed., New York: McGraw-Hill.

TREYNOR, J. (1965): "Towards a Theory of the Market Value of Risk Assets", manuscrito no publicado.

UYEMURA, D. G. y VAN DEVENTER, D. R. (1993): “Financial Risk Management in Banking”, Bankers Publishing Company \& Probus Publishing Company.

VALLS PEREIRA, P.L.; HOTTA, L.K.; SOUZA, L.A.R. y ALMEIDA, N. M. C. G. (1999): "Models to Extract the Volatility of Assets: A Comparative Study", The Brazilian Review of Econometrics, Vol. 19, n 1, Maio, pp. 57-110.

VILARIÑN SANZ, Á. (2001): Turbulencias Financieras y Riesgos de Mercado. Madrid: Pearson Educación, S. A.

WESTON, J. F. y COPELAND, T. E. (1995): Managerial Finance. 9a ed., New York, McGraw-Hill Companies, 1992 (tr. española de Jaime Gómez Mont y revisión técnica Víctor Guarnizo, "Finanzas en Administración”, McGraw-Hill, México 1995). 